\title{
Equilibrium Constraints Applied to RMF-Driven Compact Toroid Devices
}

\author{
Kenneth F. McKenna
}
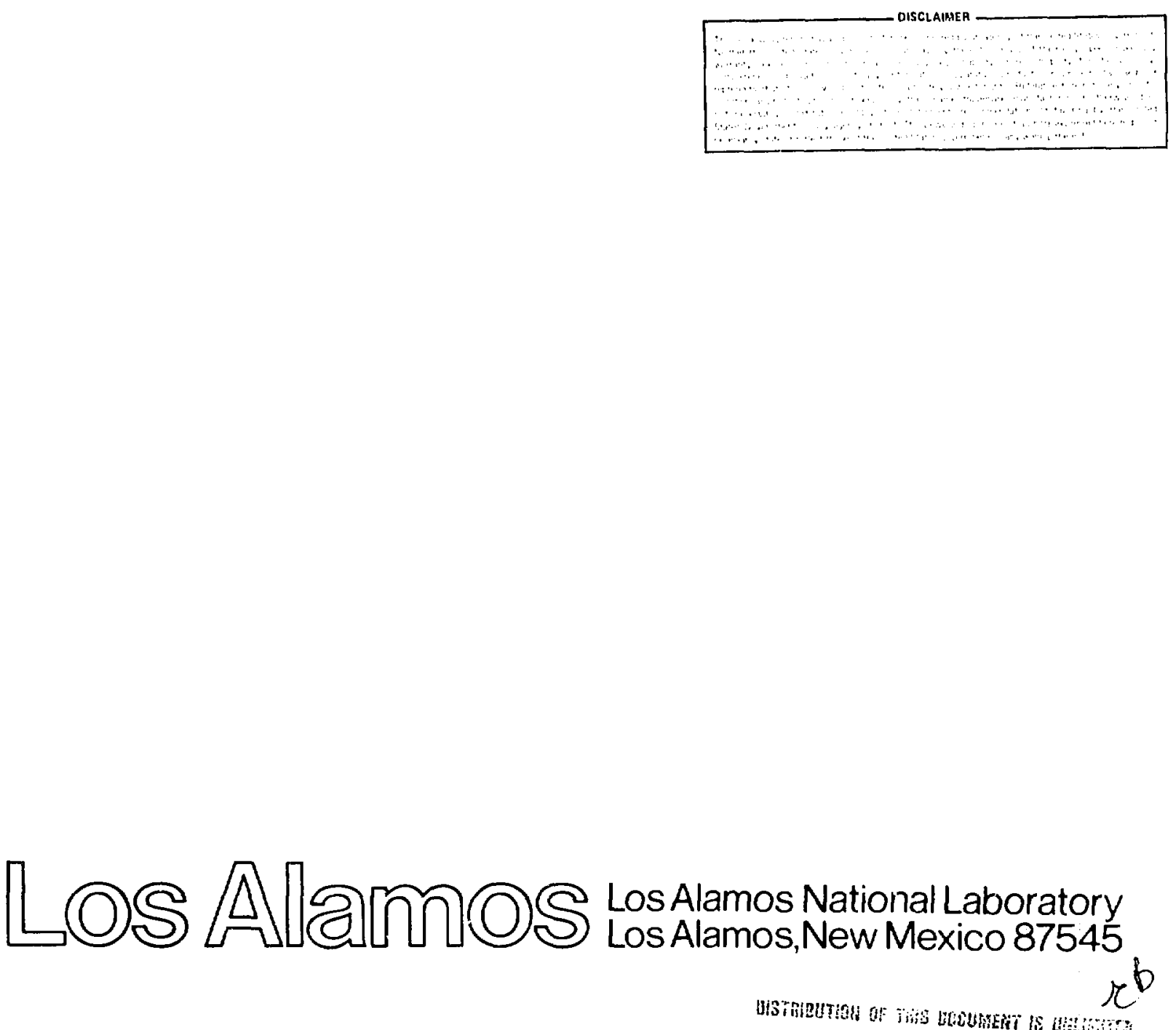
EQUILIBRIUM CONSTRAINTS APPLIED TO RMF-DRIVEN COMPACT TOROID DEVICES

by

Kenneth F. McKenna

\begin{abstract}
The rotating-magnetic-field technique is being considered as a possible means of generating and sustaining a field-reversed configuration (FRC). In this report analytical expressions for the time evolution of the rotating-field and ohmically-heated-plasma parameters are derived for a loss-less equilibrium FRC. The parameters for proof-of-concept and reactor-size devices are presented.
\end{abstract}

I. INTRODUCTION

The rotating-magnetic-field (RMF) technique has been suggested as a means of generating and sustaining a compact toroid (CT) configuration. A review of the RMF concept, its basic principles, historical development, and current theoretical understanding has been presented in a previous report. ${ }^{2}$

Figure 1 schematically illustrates the application of the RMF technique to the case of a field-reversed configuration (FRC), a CT plasma without toroidal magnetic field. The following physical description of the generation of the FRC by the rotating field is based on the theoretical analysis of Blevin and Thonemann. 3 The transverse rotating magnetic field of magnitude $B_{O}$, which is applied along the length, $\ell$, of the FRC, can be simply produced by two dipole colls that carry currents of the same frequency, and amplitude but differ in phase by $90^{\circ}$. When the angular frequency, $w$, of the rotating field lies between the electron, $\omega_{c e}$, and $i o n, \omega_{c i}$, cyclotron frequencies, calculated with respect to $B_{O}$, then the electrons follow the motion of the rotating field, resulting in the generation of an azimuthal current, while the ions lag behind. When the electron-ion collision frequency, $v_{e i}$, is much less than $\omega_{c e}$, the electrons are tightiy tied to the rotating-field lines and the 
electron current density attains its maximum value, $f_{\theta} *$ newr. If no competing mechanisms exist, electron-1on collisions would ultimately bring the lons into synchronous rotation with the electrons and the current would subsequently vanish.

The FRC can be produced in the presence of an externally applied, steady axial magnetic field, $B_{a}$. The RMF-driven azimuthal current produces an axial magnetic field, $B_{2}$, which can cancel the applied field, leading to the field-reversed configuration. When the azimuthal current is sufficient to produce $B_{z}(r=0) \cong-B_{a}$, then the FRC separatrix radius, $r_{s}$, is approximately equal to the wall radius, $r_{w}$. This limiting case of $r_{a}=r_{w}$ car also be generated by eliminating the applied field, $B_{a}$, and by substituting a discharge tube wall that is electrically conducting in the azimuthal direction, i.e., a flux-conserving wall. In this case the total magnetic flux within the discharge tube is zero before the initiation of the rotating field. When the rotating field penetrates the plasma the resulting azimuthal electron current produces a magnetic flux that is completely canceled by an equal flux generated by the flux-conserving wall current, thus producing an FRC with $\mathbf{r}_{\mathbf{S}}=\mathbf{r}_{\mathbf{w}}$.

Blevin and Thonemann ${ }^{3}$ have carried out the steady-state analysis of an infinitely long, RMF-driven FRC plasma. They consider a uniform (In space and time) temperature FRC sustained by a transverse rotating magnetic field, where $\omega_{c i}<\omega<\omega_{c e}$ Assuming that the lons are stationary, the appropriate Ohm's law is

$$
E=\frac{1}{n e} j \times \underset{B}{ }+n j-\frac{1}{n e} \nabla p_{e}
$$

where $n$ and $n$ are the plasma density and resistivity, respectively. With the rotating magnetic field of the form,

$$
\begin{aligned}
& B_{r}=B_{0} \cos (\omega t-\theta) \\
& B_{\theta}=B_{0} \sin (\omega t-\theta),
\end{aligned}
$$


and an applied bias field, $B_{a}$, the solution of Eq. (1) for the azimuthal current density is

$$
j_{\theta}=\frac{\text { newr }}{1+2\left(\frac{\nu_{\mathrm{ei}}}{\omega_{c e}}\right)^{2}} \text {. }
$$

For $\nu_{e i}$ 'wce much less than unity, the "slip" between the electron gas and the rotating field is small. In this event, a rigid-rotor current density distribution results

$$
j_{\theta}=\text { newr, }
$$

and the electrons are perfectly "tied" to the rotating magnetic field lines. From the equilibrium condition

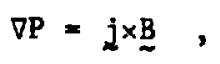

it can be shown [with $T \neq T(r)$ ] that the equilibrium particle density and magnetic field profiles are given by

$$
\begin{aligned}
& n(r)=n_{m} \operatorname{sech}^{2} k\left(\frac{r^{2}}{r_{0}^{2}}-1\right), \\
& B_{z}(r)=B_{a} \tanh K\left(\frac{r^{2}}{r_{o}^{2}}-1\right),
\end{aligned}
$$

where $r_{0}$ is the magnetic axis, $B_{z}\left(r_{0}\right)=0$, and $n_{m}$ is the density at $r=r_{0}$. The factor $K$ has the value 


$$
K=\tanh ^{-1}\left(\frac{B_{z}(0)}{B_{a}}\right) .
$$

The separatrix radius, $r_{s}$, is defined by the equation

$$
\int_{0}^{r_{s}} 2 \pi B_{2}(r) d r=0,
$$

so that from Eq. (7) [or any function of the form $f\left(\frac{r^{2}}{r_{0}^{2}}-1\right)$ ],

$$
r_{s}=\sqrt{2} r_{0}
$$

Solutions to the initial-value problem, in which a transverse rotating magnetic field is suddenly applied to a collisional ( $\omega<v_{\text {ef }}$ ) plasma column of uniform temperature and density, have been obtained numerically. ${ }^{4,5}$ As in the steady-state analysis, the nonlinear Ohm's law, Eq. (1), was used, ion motion was neglected, and the condition $\omega_{c i}<\omega<\omega_{c e}$ was applied. The results show that, for $v_{e i} / \omega_{c e}<1$, the rotating field fully penetrates the plasma when

$$
\frac{\nu_{e 1}}{\omega_{c e}} \frac{r_{w}}{\delta} \leq 1,
$$

where $\delta$ is the classical skin depth. The rotating magnetic field does not instantaneously penetrate the plasma but rather diffuses into the plasma in a time given by 5

$$
\tau_{d}=\frac{1}{8} \frac{\mu_{0} r_{w}^{2}}{\eta} .
$$


The above discussion outlines the basic theoretical principles of the RMF technique. Experimentally, it has been demonstrated in $\operatorname{small}\left(\mathrm{r}_{\mathrm{w}}<10 \mathrm{~cm}\right)$ low temperature ( $T_{e}<50 \mathrm{eV}$ ) collisional plasma devices that this technique can be used to generate a variety of plasma-field geometries such as RFP, 6 screw pinch, ${ }^{7}$ and FRC. $3,8,9$ The objective of these previous experiments was to verify that the rotating magnetic field could, under the appropriate conditions, penetrate the plasma and subsequently drive the current required for the desired magnetic-field configuration. The configuration lifetime has generally been limited by the rotating-field power supplies to a few tens of microseconds. Accordingly, the maintenance of an equilibruim configuration by proper programming of the applied fields was not necessary in previous short-lived experiments. However, in considering the applicability of the RMF technique to the production of large $\left(r_{w} \sim 100 \mathrm{~cm}\right)$ long-lived (milliseconds) FRC plasmas of fusion interest the maintenanse of plasma equilibrium is of primary importance. In this report equilibrium constraints are applied to the analysis of RMF-generated FRC plasmas. MKS units are used.

\section{RMF-GENERATED EQULIIBRIUM FRCS}

Figure 1 shows an RMF-generated and -sustained FRC. The plasma is heated ohmically and can be maintained in pressure balance by proper programming of the equilibrium current. Because the plasma is assumed to exist for long times, programmed puff-gas injection is also considered. The objective of the following analysis is to obtain analytical expressions for the time evolution of the plasma and RMF parameters. Using these expressions, the scaling of the RMF technique to FRCs of fusion interest can be estimated.

To simplify the analysis, the FRC is assumed to be contained within a flux-conserving wall with no applied blas field. Hence, $r_{s} \simeq r_{w}, B_{a} \cong B_{w}$, and Eqs. (6) and (7) can be used to yield $n_{m}=2 n_{0}$, where $n_{0}$, is the fill density, and $K$ has been taken as $K=2$. It is further assumed that the fons remain motionless with $T_{1} \simeq 0$, and that $T_{e} \neq T_{e}(r)$.

Considering the equilibrium FRC, the electron population energy balance can be written as

$$
\left.\left.\frac{d W}{d t}=\frac{d W}{d t}\right)_{\text {ohmic }}-\frac{d W}{d t}\right)_{10 s s},
$$


where $W$ is the total electron thermal energy,

$$
w=\frac{3}{2} \ln r_{w}{ }^{2} n_{0} k T_{e} \text {. }
$$

The loss term in Eq. (13) accounts for energy losses through electron-ion equilibration, radiation, and particle and energy transport. All these loss processes will have a significant affect on the time development of the FRC. However, in order to obtain "zeroth-order analytical solutions," all losses are neglected so

$$
\left.\frac{d W}{d t}\right)_{10 s s}=0 .
$$

The ohmic heating term in Eq. (13) is

$$
\left.\frac{d W}{d t}\right)_{\text {ohmic }}=\int_{0}^{2 \pi} \int_{0}^{r_{W}} n j_{\theta}^{2} \operatorname{lrd} \theta d r .
$$

Using Eqs. (4) and (6) with $n_{m}=2 n_{0}$ and $k=2$ yields

$$
\left.\frac{d W}{d t}\right)_{\text {ohmic }}=n \frac{32 \pi}{\ell}\left(\frac{e \omega n_{o} \ell r_{w}^{2}}{2}\right)^{2} \phi,
$$

where

$$
\phi=\int_{0}^{1} \operatorname{sech}^{4}\left[2\left(2 x^{2}-1\right)\right] x^{3} d x .
$$

From numerical integration, $\phi \simeq 0.09$. The total RMF-driven current is 


$$
\begin{aligned}
I_{\theta} & =\int_{0}^{r_{w}} j_{\theta} \ell d r \\
& =\frac{e w n_{0} \ell r_{w}^{2}}{2},
\end{aligned}
$$

so that the energy input by ohmic heating is

$$
\left.\frac{d w}{d t}\right)_{\text {ohmic }}=n \frac{3 \pi}{\ell} I_{\theta}^{2}
$$

The equilibrium current, It, can be related to the plasma density and temperature through the pressure balance condition and Ampere's law. Pressure balance requires

$$
n_{m} k T_{e}=\frac{B_{w}^{2}}{2 \mu_{o}}
$$

and Ampere's law gives

$$
B_{w}=\frac{\mu_{o} I_{\theta}}{2 l}
$$

Combining Eqs. (21) and (22) and substituting in Eq. (20) ylelds

$$
\left.\frac{d W}{d t}\right)_{\text {ohmic }}=n \frac{46 \pi 2 n_{0} k T_{e}}{\mu_{0}} .
$$


With

$$
\eta=5.4 \times 10^{-9} \frac{\ell n \Omega}{T_{e}{ }^{3 / 2}}(\Omega-m),
$$

where $T_{e}$ is in keV and $\ell n \Omega$ is taken as 10 , and allowing the fill density to be time dependent (puff injection), Eqs. (24), (23), and (14), can be substituted into Eq. (13) to yield

$$
\mathrm{T}_{\mathrm{e}}{ }^{1 / 2} \frac{\mathrm{dT} \mathrm{T}_{\mathrm{e}}}{\mathrm{dt}}+\frac{\mathrm{T}_{\mathrm{e}}^{3 / 2}}{\mathrm{n}_{\mathrm{o}}} \frac{\mathrm{dn_{o }}}{\mathrm{dt}}=\frac{1.3}{\mathrm{r}_{\mathrm{w}}^{2}} .
$$

A relation between $n_{0}$ and $T_{e}$ is required in order to obtain a solution to the above equation. This relation is obtained from a consideration of the expression for the RMF-generated currenc and the pressure balance condition. From Eq. (19)

$$
I_{\theta} \propto \omega n_{0}
$$

and from Eqs. (21) and (22)

$$
\mathrm{I}_{\theta}^{2} \propto \mathrm{n}_{\mathrm{o}} \mathrm{T}_{\mathrm{e}}
$$

The equilibrium current is programmed by varying both $w$ and $n_{0}$ in time. Allowing

$$
\omega \alpha n_{0}^{\gamma} \text {, }
$$

Eqs. (26) and (27) yield 


$$
n_{0} \propto T_{e}^{\frac{1}{2 \gamma+1}}
$$

Thus, using Eq. (29), Eq. (25) becomes

$$
\frac{2(\gamma+1)}{2 \gamma+1} \mathrm{~T}_{e}{ }^{1 / 2} \frac{\mathrm{dT}}{\mathrm{d} \mathrm{e}_{\mathrm{e}}}=\frac{1.3}{\mathrm{r}_{\mathrm{w}}^{2}} \text {, }
$$

which can be immediately integrated to yield

$$
T_{e}=\left[\frac{3}{2} \frac{2 \gamma+1}{2(\gamma+1)} \frac{1.3 t}{r_{w}^{2}}+T_{o}^{3 / 2}\right]^{2 / 3}(\mathrm{keV}),
$$

where $T_{0}$ is the plasma temperature at $t=0$. The plasma fill density and the RMF angular trequency are given by

$$
\begin{aligned}
& n_{0}(t)=n_{0}(0)\left(\frac{T_{e}}{T_{0}}\right) \frac{1}{2 \gamma+1}, \\
& \omega(t)=\omega(0)\left(\frac{T_{e}}{T_{0}}\right) \frac{\gamma}{2 \gamma+1},
\end{aligned}
$$

where $n_{0}(0)$ and $\omega(0)$ are the fill density and rotating-field angular frequency, respectively, at time $t=0$. It should be noted that as $\gamma+\infty$, $n_{0}(t) \rightarrow$ constant and $w \alpha T_{e}^{1 / 2}$. This is the case without puff-gas injection. The plasma configuration is maintained by programming only the rotating-field frequency. This could lead to technological difficulties when attempting to drive the FRC from a few eV into the keV range. The lower bound on $\gamma$ is determined from a consideration of the parameters required for penetration and sustainment within the plasma of the rotating field. 
As previously discussed, numerical results 4,5 show that the ratating field completely penetrates the initial plasma if the penetration parameter, $\left(v_{e i} / \omega_{c e}\right)\left(r_{w} / \delta\right)$, is less than or about equal to unity. This result is supported experimentally. $3,8,9$ The rotating field is observed to remain within the plasma as long as the penetration condition is satisfied. When $\left(v_{e i} / \omega_{c e}\right)\left(r_{r y} / \delta\right)>1$, the rotating field is no longer sustained within the plasma and the azimuthal current resistively decays. The penetration parameter can be written as

$$
\frac{v_{\text {el }}}{\omega_{\text {ce }}} \frac{r_{w}}{\delta}=9.2 \times 10^{-27} \sqrt{\ell n \Omega} \sqrt{\omega} \frac{n_{0}}{B_{0}} \frac{r_{w}}{T_{e} e^{3 / 4}} .
$$

The rotating-field angular frequency, $w$, and rotating-fleld amplitude, $B_{o}$, are related through the required condition

$$
\omega_{\text {ci }}<\omega<\omega_{\text {ce }},
$$

where

$$
w_{c i}=\frac{e B_{0}}{m_{1}} .
$$

From the Davenport et al. 9 experiments in hydrogen, the lower bound on $w$ can be taken as

$$
\omega=5 \omega_{c i} \text {. }
$$

Taking $\left(v_{e 1} / w_{c e}\right)\left(r_{w} / \delta\right)=1$, ln $\Omega=10$, and substituting Eq. (37) in Eq. (34) yields for deuterium 


$$
7 \times 10^{-18} \frac{\mathrm{n}_{\mathrm{o}} \mathrm{r}_{\mathrm{w}}}{\sqrt{\omega} \mathrm{T}_{\mathrm{e}}^{3 / 4}}=1,
$$

which must be satisfied for the entire duration of the RMF current drive.

The plasma heating and density redistribution processes occurring during the FRC formation phase, when the rotating field is penetrating the initial plasma and the azimuthal current is developing, have not been theoretically or experimentally investigated. For simplicity, the formation phase is neglected in this analysis and the plasma is assumed to be in equilibrium and to heat uniformly from time $t=0$. Under this assumption the time evolution of the plasma variables in the penetration parameter, Eq. (38), is known from Eqs. (31), (32), and (33). Accordingly, the penetration parameter can be written as

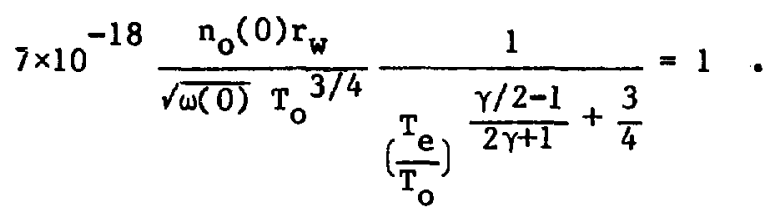

Several interesting conclusions can be extracted from an examination of this expression. For $\gamma<1 / 8$, the exponent on $\left(\mathrm{T}_{\mathrm{e}} / \mathrm{T}_{0}\right)$ is negative so that if the equation is satisfied for $t=0$ it will not be satisfled for any $t>0$, and the rotating field will not be sustained within the plasma. At $\gamma=1 / 8$, the exponent goes to zero and if the equation is satisfied at $t=0$, it will be satisfied for all $t$. This lower limit on $\gamma$ gives the slowest rate of Increase of $\omega, \omega \propto T_{e} e^{1 / 10} \propto t^{1 / 15}$, and the fastest rate of increase of $n_{0}, n_{0}$ $\alpha \mathrm{T}_{\mathrm{e}}^{4 / 5} \alpha \mathrm{t}^{8 / 15}$, In order to malntain equilibrium. For $\gamma>1 / 8$, the left-hand side of Eq. (39) decreases with time, better satisfying the condition, $\left(\nu_{e 1} / \omega_{c e}\right)\left(r_{w} / \delta\right)<1$. Accordingly, the bounds on $\gamma$ are

$$
1 / 8<\gamma<\infty .
$$


At $t=0, T_{e}=T_{0}$ so the penetration condition, Eq. (39), yields, for the initial rotating-field frequency,

$$
\omega(0)=4.9 \times 10^{-35} \frac{n_{0}(0)^{2} r_{w}^{2}}{r_{0}^{3 / 2}}(\mathrm{rad} / \mathrm{sec}) .
$$

An Independent expression for $\omega(0)$ is obtained by combining the pressure balance condition, $\mathrm{Eq} .(21)$, and the result of the application of Ampere's law, Eq. (22), which gives the equilibrium current

$$
\mathrm{I}_{\theta}^{2}=\frac{16 \ell^{2} \mathrm{n}_{\mathrm{o}} \mathrm{kT} e}{\mu_{\mathrm{o}}}
$$

and substituting this expression ini:o the total RMF-driven current, Eq. (19), evaluated at $t=0$,

$$
w(0)=5.6 \times 10^{14} \frac{T_{o}^{1 / 2}}{r_{w}^{2} n_{o}(0)^{1 / 2}}(\mathrm{rad} / \mathrm{sec})
$$

The required inftial fill density is then obtained by eliminating $w(0)$ between Eqs. (41) and (43),

$$
n_{0}(0)=4.2 \times 10^{19} \frac{T_{0}^{4 / 5}}{r_{w}^{8 / 5}}\left(m^{-3}\right)
$$

Thus, for a given initial plasma temperature and discharge tube wall radius, $\mathrm{n}(0)$ is determined from Eq. (44), w(0) from Eq. (41), and the time evolution of $T_{e}, n_{0}$, and $w$ from Eqs. (31), (32), and (33), respectively. 
Note that the initial conditions can be obtained without assuming that the plasma is in equilibrium from time $t=0$. For example, the rotating field can be assumed to diffuse into the initial plasma at constant fill density and rotating-field frequency, uniformly heating the inttial plasma during the diffusion phase. The required system parameters can then be calculated at the end of the diffusion time, based on the plasma temperature at that time. Under these assumptions, the expressions for $\omega\left(\tau_{d}\right)$ and $n_{o}\left(\tau_{d}\right)$ are of the same functional form as Eqs. (41) and (44), but with slightly different constants.

In applying the RMF technique to the generation of FRC plasmas it is of interest to determine the rate at which energy is absorbed in the plasma by ohmic heating. From Eqs. (23), (24), and (32) this rate can be expressed as

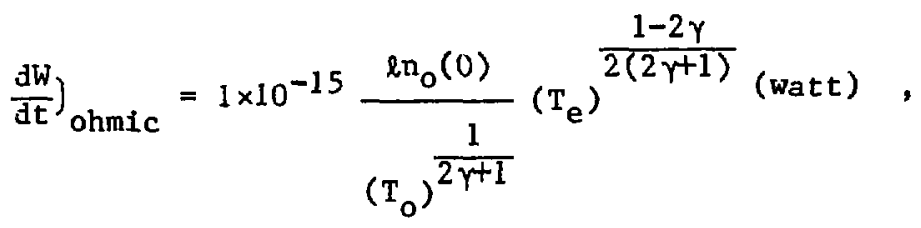

where the time dependence of $T_{e}$ is given by Eq. (31). Note that for $1 / 8<\gamma<1 / 2$, the ohmic power input increases as a function of time, whereas for $1 / 2<\gamma<\infty$ the power input decreases as a function of time. This effect is directly attributable to the allowed programming of the gas fill density.

In the above analysis the plasma ions have been assumed to be energetically decoupled from the electrons with $T_{1} \simeq 0$ for all $t$. In an actual system, the ions will gain energy from the electrons on an equilibration time scale given by

$$
\tau_{\text {eq }}=2 \times 10^{19} \frac{T_{e^{3 / 2}}}{n_{o} \ln \Omega}(\mathrm{sec})
$$

In addition, electron-ion collisions could ultimately bring the ions into rotation with the electrons, resulting in a cancellation of the RMF-driven current. The characteristic time for this process is 


$$
\begin{aligned}
T_{s}= & 2 \sqrt{2} \frac{m_{i}}{m_{e}} \tau_{e e} \\
& =5.8 \times 10^{19} \frac{T_{e}^{3 / 2}}{n_{o} \ln \Omega}(\mathrm{sec}) .
\end{aligned}
$$

Finally, the voltage required io drive the rotating magnetic field can be estimated assuming that the rotating field is produced by two sets of dipole coils that carry currents of equal frequency and magnitude but differ in phase by $90^{\circ}$. The voltage appearing across each dipole loop is

$$
V=B_{0} A \omega(v 01 t)
$$

where $A$ is the area defined by the loop. This expression neglects geometry effects and power transfer to the plasma. The actual required voltage could be several times higher than indicated by Eq. (48). Thus, this expression sets a lower bound on the dipole loop voltage.

III. PARAMETERS OF TWO RMF-DRIVEN FRC DEVICES

In order to illustrate the magnitudes and time variations of the plasma and RMF parameters, the relations given in the previous sections are applied to a $0.2-m$-radius and a 1.0-m-radius FRC device. The smaller device, which has dimensions similar to that of FRX-C, 10 can be considered a proof-of-concept experiment. The larger-radius machine would be typical of reactor-size devices.

In both cases the parameters are given assuming an initial $(t=0)$ fully ionized plasma of $2 \mathrm{eV}$ temperature. Curves are presentd for $\gamma=\infty$ (constant fill density, and fastest rate of increase of rotating-field frequency) and for $\gamma=1 / 8$ (puff-gas injection at fastest possible rate, and slowest rate of increase of rotating-field frequency). The calculations are carried out to $t=0.1 \mathrm{sec}$. 
Figure 2 shows the time history of the plasma electron temperature for the RMF-driven equilibrium FRC. Again, the lon temperature is assumed to be zero for all time $t$. As can be seen, the smaller device heats much more rapidly than the larger device. For fixed radius, maximum puff-gas injection lowers the temperature obtained at constant fill density by a factor of about $2 / 3$.

Figures 3, 4, 5, and 6 show the time development of the fill density. To 11lustrate the early time behavior, Figs. 4 and 6 are plotted from $t=0$ to $t=1 \mathrm{msec}$. The smaller device operates at significantly higher densities and at a faster rate of fill density increase than the larger machine.

The time evolution of the rotating-field frequency is given in Figs. 7, 8, 9, and 10. Again the smaller machine exhibits larger values of the time-dependent parameter than the larger device. Puff-gas injection significantly lowers the required equilibrium rotating-field frequency, a technological advantage.

Figures 11 and 12 give the time evolution of the axial magnetic field at the wall, generated by the flux-conserving wall currents, and magnitude of the transverse rotating field. As a result of the higher temperature and densities obtained in the $0.2-m$-radius device, the confining field at the wall in this device is much larger than the confining field in the 1-m-radius machine, at the same value of $t$. The ratio $B_{w} / R_{0}$ significantly increases with time in the case with puff-gas infection.

In calculating the voltage applied to the dipole-coll set that produces the rotating field, the machine length has been taken as equal to three times the wall radius. The coll voltage is given in Figs. 13 and 14 . The required voltage is highly dependent on the fill density variation, being much lower with puff injection. The voltage decreases significantly with increasing machine radius, another technological advantage.

Figures 15 and 16 give the rate of energy deposition, by ohmic heating, into the electron plasma. The interesting point here is that puff-gas injection, for values of $\gamma>1 / 2$, results in a continual increase in energy input as a function of time even in the presence of increasing electron temperature.

Figures 17 and 18 present the time history of the time required to bring the lons into rotation with the electrons, $\tau_{s}$, and the electron-ion equilibration time, $\tau_{e q}$, for the case of maximum puff-gas injection. Both $\tau_{B}$ 
and $\tau_{\text {eq }}$ increase as $r_{w}$ increases, at a given time $t$, primarily owing to the lower densities. For both size devices, $\tau_{s}$ and $\tau_{e q}$ are short compared to the $0.1 \mathrm{sec}$ extent of the calculation time. For $\gamma=\infty$ (constant density), $\tau_{s}$ and $\tau$ ea are always less than $t$ so that these effects can be neglected in this case. Clearly, electron-ion equilibrium and fon spin up must be included in a detalled analysis of the equilibrium state obtained in the more interesting case of puff-gas injection.

\section{RECOMMENDATION FOR FIJTURE WORK}

The present work should be regarded as a "zeroth-order" analysis of the implications of equilibrium constraints on the time development of an RMF-driven FRC. These constraints have not been previnusly considered in the theoretical treatment of the RMF concept. Recause no absolutely fatal flaws in the application of the RMF technique to the generation and sustainment of FRCs have been uncovered as a result of the present work, a more detalled analysis of this technique appears justifled. Discussed below are a few of the areas that should be considered for inclusion in future work.

The initial FRC formation phase has been completely neglected in the present work where an equilibruim FRC is assumed to exist from time $t=0$. Future analysis could include a treatment of the initial set-up phase, although little guidance can be obtained from past experiments. Theory indicates that the rotating field diffuses into the initial preionized plasma, thus making the formation process dependent on the plasma resistivity. The effect of anomalous resistivity on this process should be investigated. Assuming that the FRC is heated ohmically, anomalous resistivity would also significantly influence the plasma temperature and could easily be included in future analysis.

An obvious improvement in the present analysis would be the addition of ion spin-up and energy-loss terms to the zero-dimensional, time-dependent electron energy balance equation. Line radiation from carbon and oxygen, which would probably be the dominant impurities, should be included. Electron energy loss by electron-ion equilibration should also be introduced in the analysis. The processes involved in particle and energy transport in FRCs are not well understood and inclusion of these effects would require the use of empirical formulas. Thermal transport in RMF-driven FRCs is further complicated by the fact that the transverse rotating field results in 16 
"open-field Iines" that intersect the wall. The effect of these time-varying open lines on the FRC energy halarice is, at present, completely unknown. 


\section{REFERENCES}

1. I. R. Jones, "The Rotamak Concept," F1inders University of South Australia report FUPH-R-151 (1979).

2. Kenneth F. McKenna, "The RMF Concept: A Rotating-Magnetic-Field Technique for Driving Steady Plasma Currents in Compact Torold Devices," Los Alamos Scientific Laboratory report LA-8516-MS (1980).

3. H. A. Blevin and P. C. Thonemann, "Plasma Confinement Using an Alternating Magnetic Field," Nucl. Fusion Suppl., Part I, p.55 (1962).

4. W. N. Hugrass, I. R. Jones, and M.G.R. Phillips, "Production of Plasma Currents Using Transverse Rotating Magnetic Fields," Flinders University of Souti Australia report FUPH-R-154 (1979).

5. W. N. Hugrass and R. C. Grimm, "A Numerical Study of the Generation of a Steady Azimuthal Current in a Plasma Cylinder Using a Transverse Rotating Radio Frequency Magnetic Field,"(to be published).

6. I. R. Jones, "On the Possibility of Generating and/or sustaining the Reversed-Field-Pinch Configuration by Means of a Transverse Rotating Magnetic Field," F1inders University of South Australia report FUPH-R-153 (1979).

7. W. N. Hugrass, I. R. Jones, and M.G.R. Phillips, "The Generation of Screw-Pinch/High-Beta-Tokamak Configurations Using a Transverse Rotating Magnetic Field," Nucl. Fusion 9, 1546 (1979).

8. W. N. Hugrass, I. R. Jones, K. F. McKenna, M.G.R. Phillips, R. G. Storer, and H. Tuczek, "Compact Torus Configuration Generated by a Rotating Magnetic Field: The Rotamak," Phys. Rev. Lett. 44, 1676 (1980).

9. P. S. Davenport, G. Frances, W. Miller, and A. F. Taylor, "Annular Plasmas Produced by Rotating Magnetic Fields," U.K.A.E.A. Culham Report CLM-R65 (1966).

10. H. Dreicer, "Proposal for FRX-C and Multiple-Cell Compact Torus Experiments," Los Alamos Scientific Laboratory report LA-8045-P (1979). 

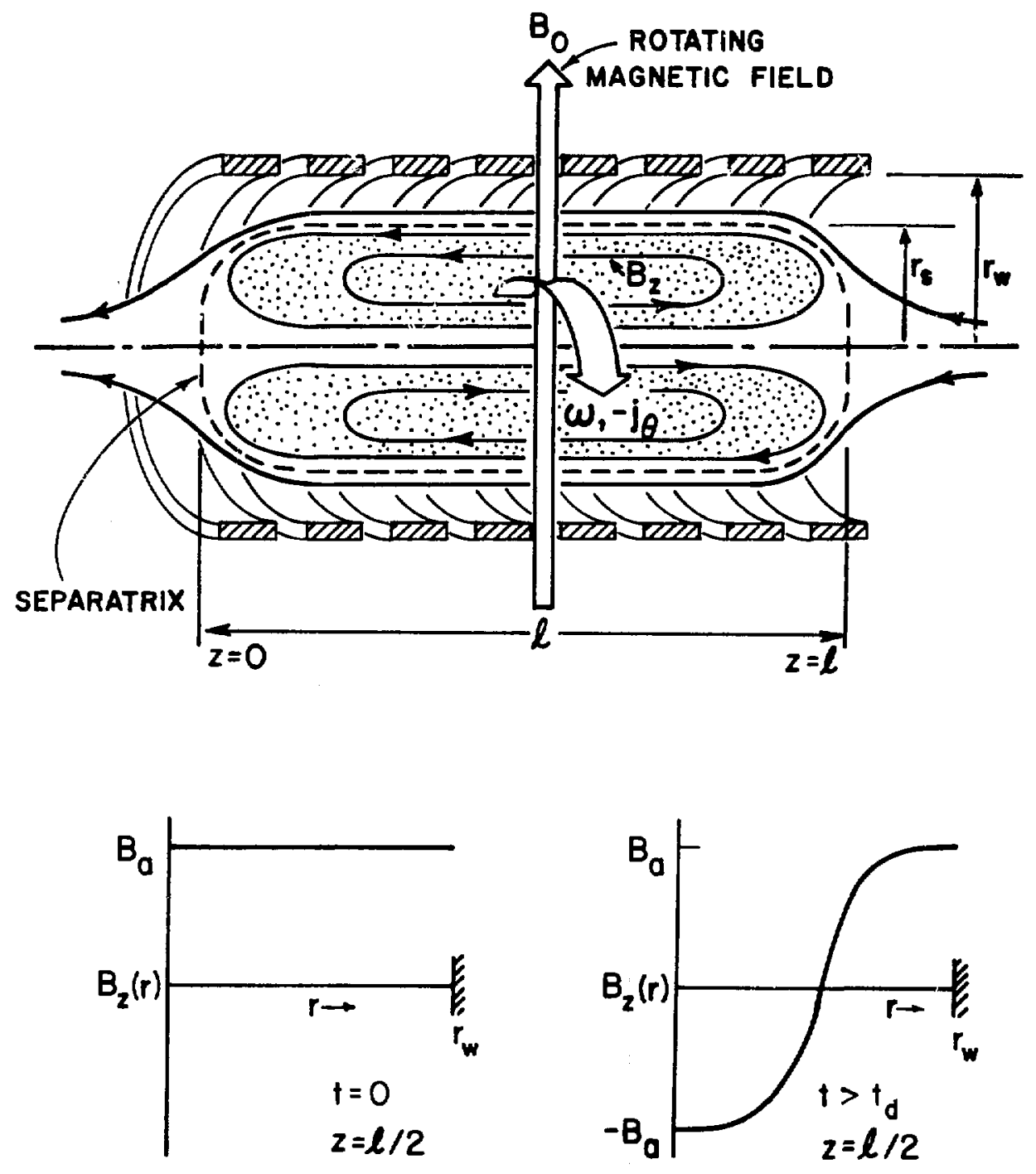

Fig. 1. Schematic RMF-driven FRC. 


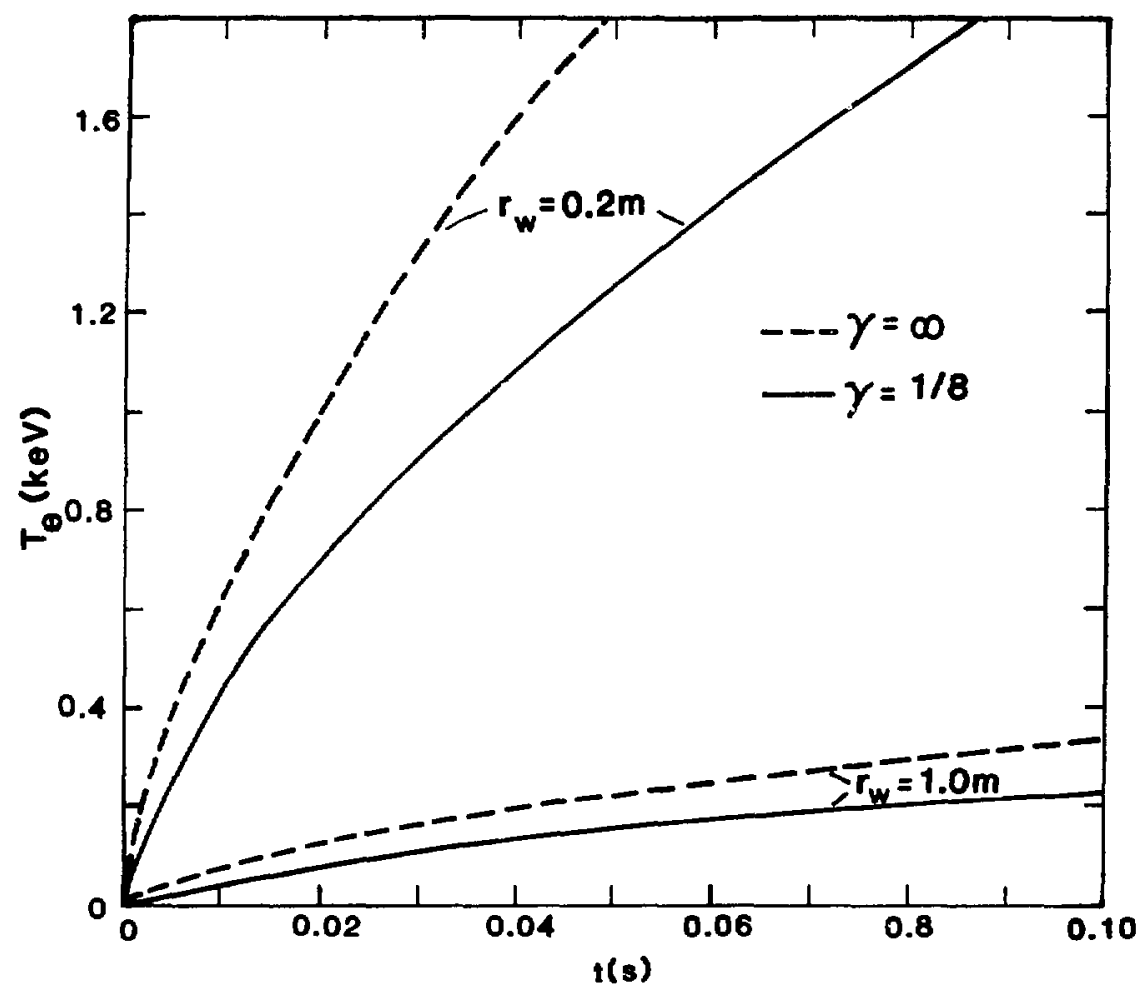

Fig. 2. Electron temperature as a function of time. 

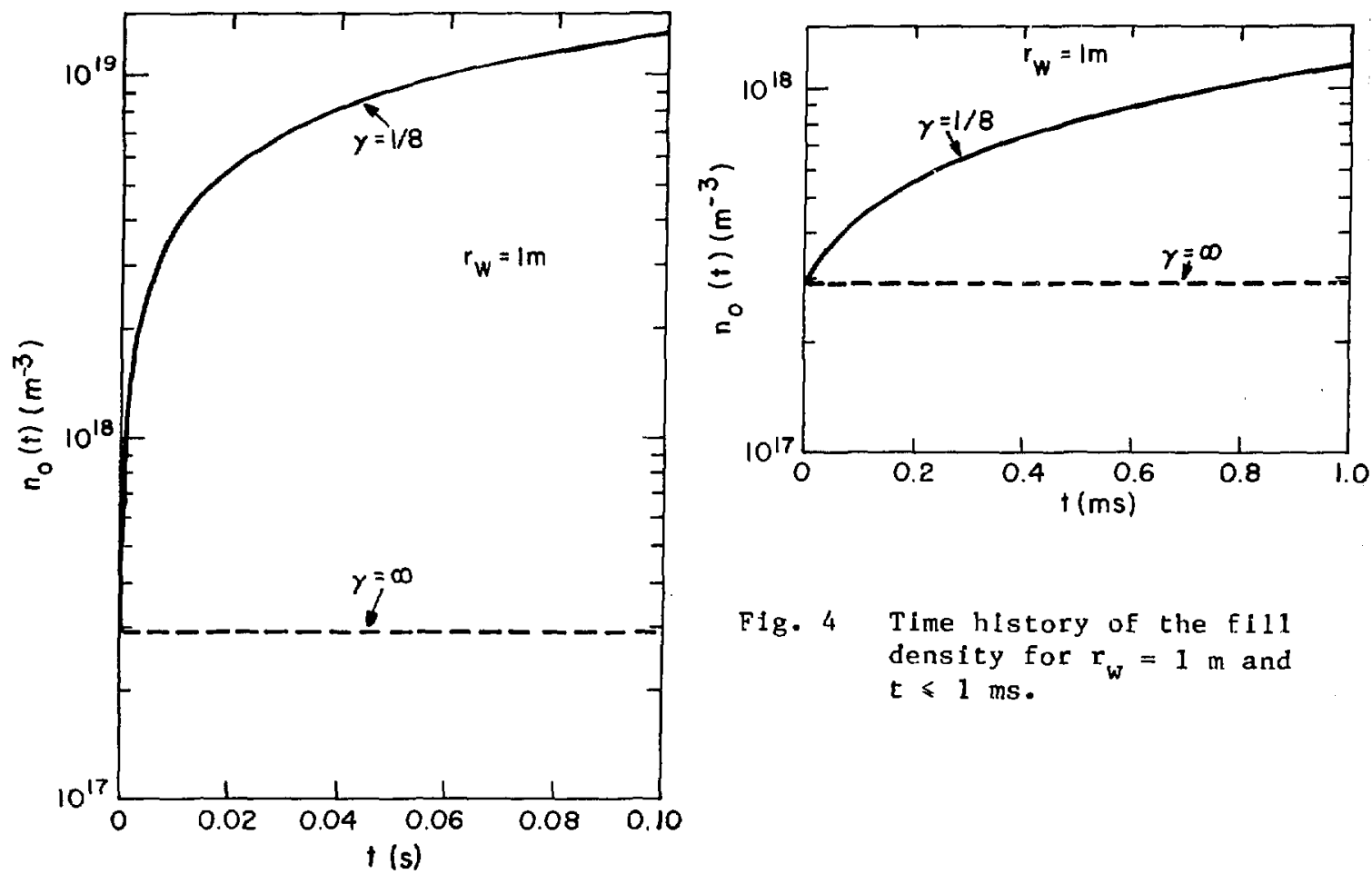

Fig. 4 Time history of the fill density for $r_{w}=1 \mathrm{~m}$ and $t \leqslant 1 \mathrm{~ms}$.

F1g. 3 Time history of the fill

density for $r_{w}=1 \mathrm{~m}$. 

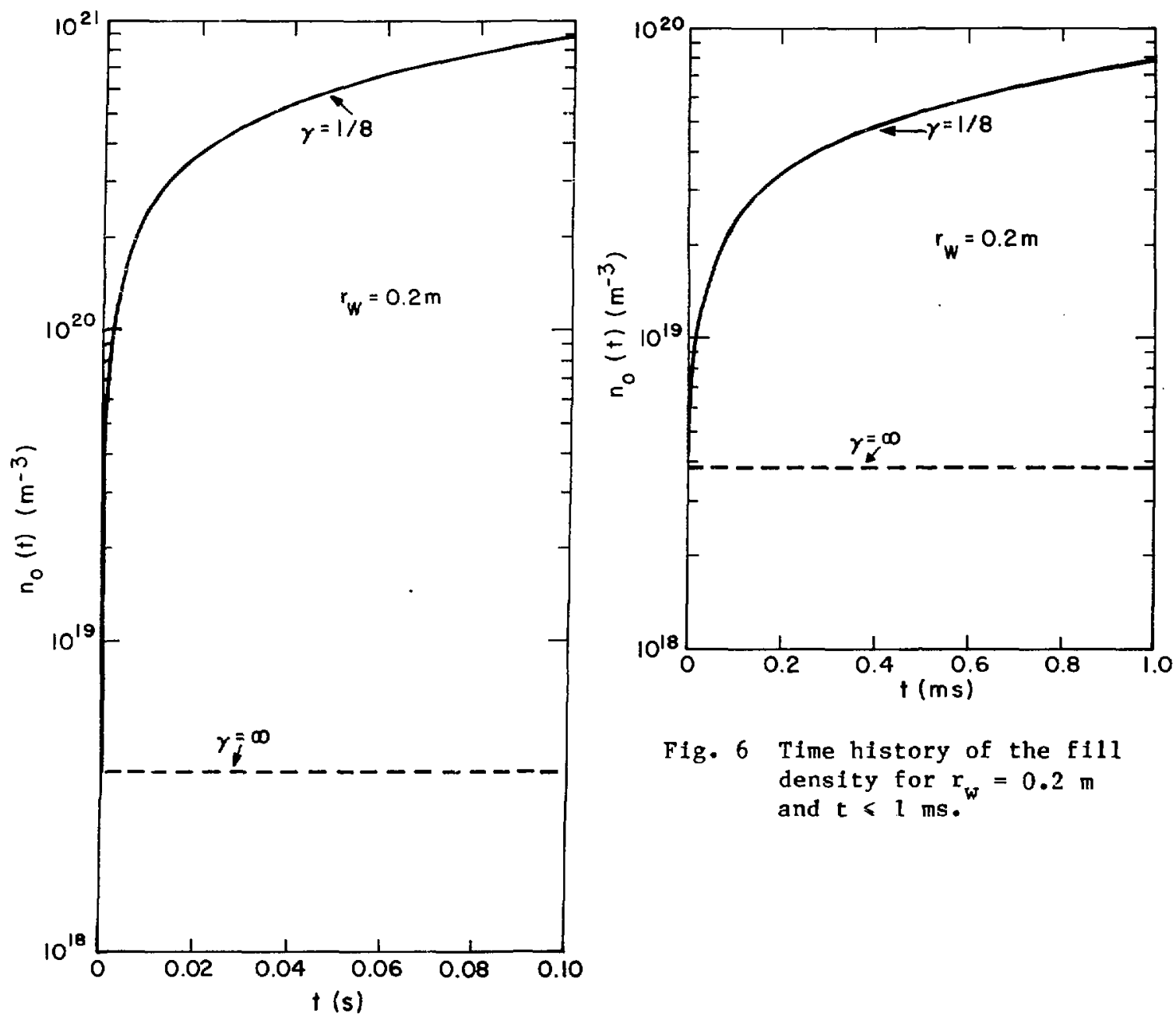

Fig. 6 Time history of the fill density for $r_{w}=0.2 \mathrm{~m}$ and $t \leqslant l$ ms.'

Fig. 5 Time history of the fill density for $r_{w}=0.2 \mathrm{~m}$. 

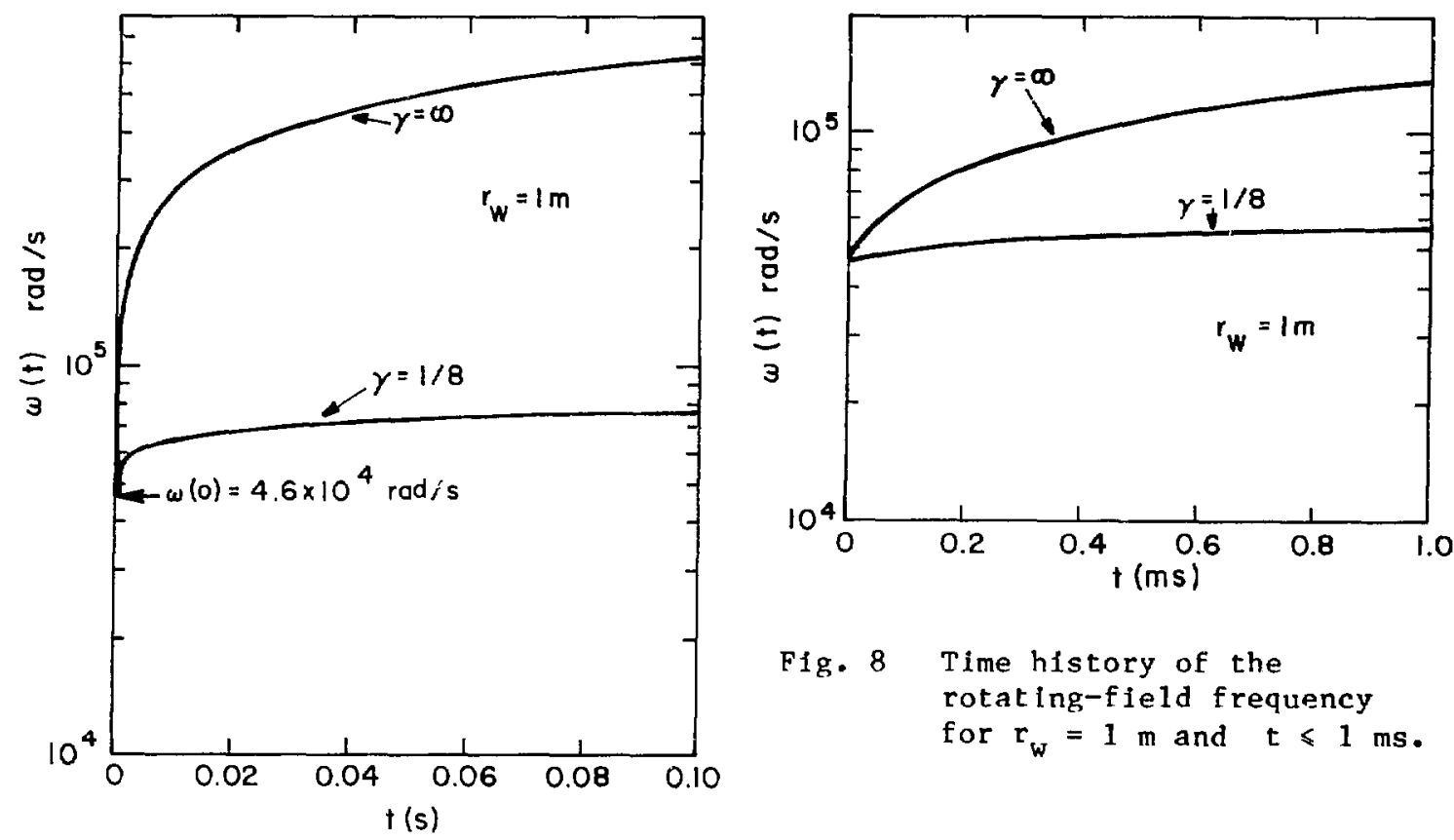

Fig. 8 Time history of the rotating-field frequency for $r_{w}=1 \mathrm{~m}$ and $t \leqslant 1 \mathrm{~ms}$.

Fig. 7 Time history of the rotating-field frequency for $r_{w}=1 \mathrm{~m}$. 

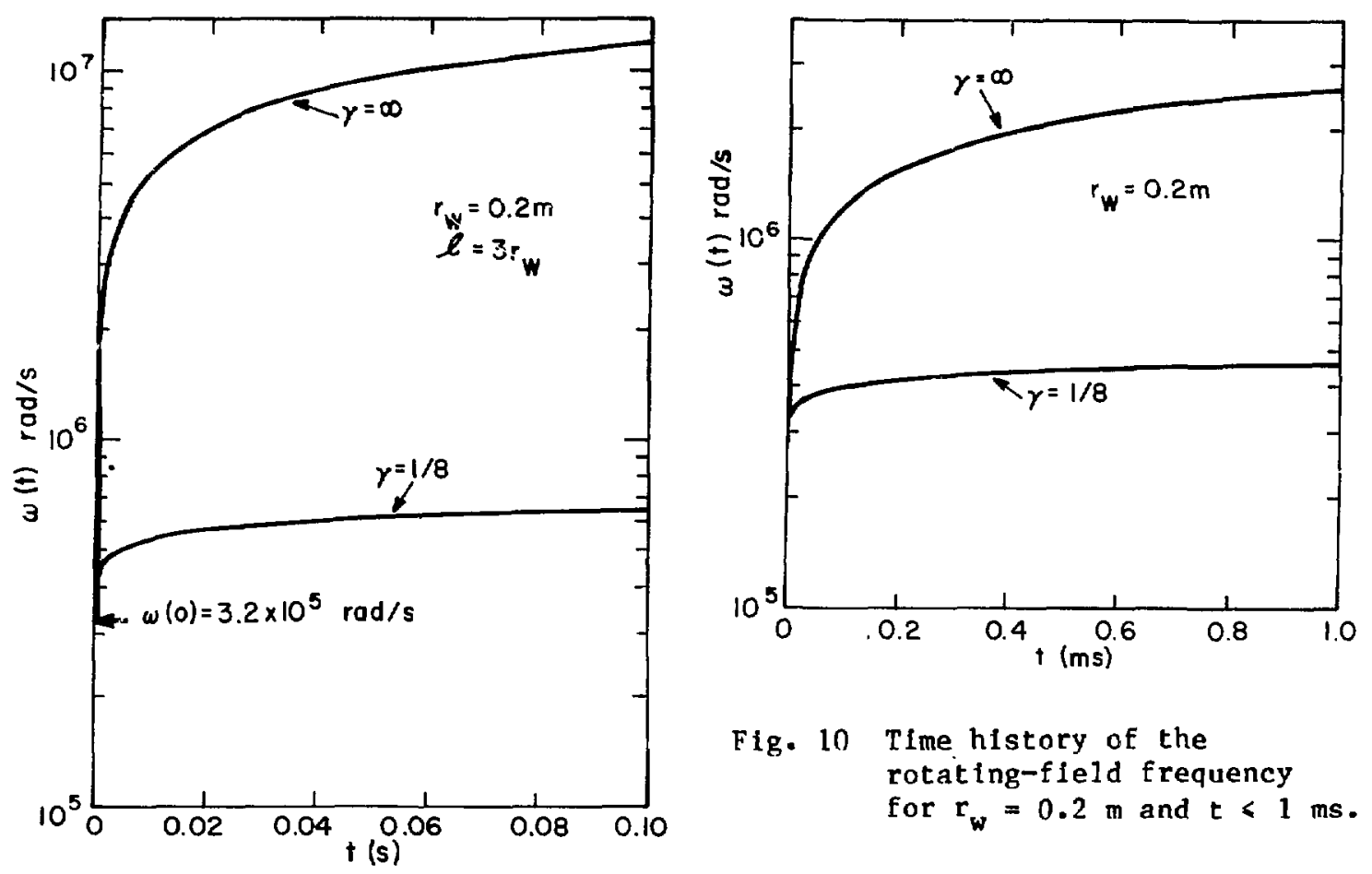

Fig. 10 Time history of the rotating-field frequency for $r_{w}=0.2 \mathrm{~m}$ and $t<1 \mathrm{~ms}$.

F1g. 9 Time history of the rotating-field frequency for $r_{w}=0.2 \mathrm{~m}$. 


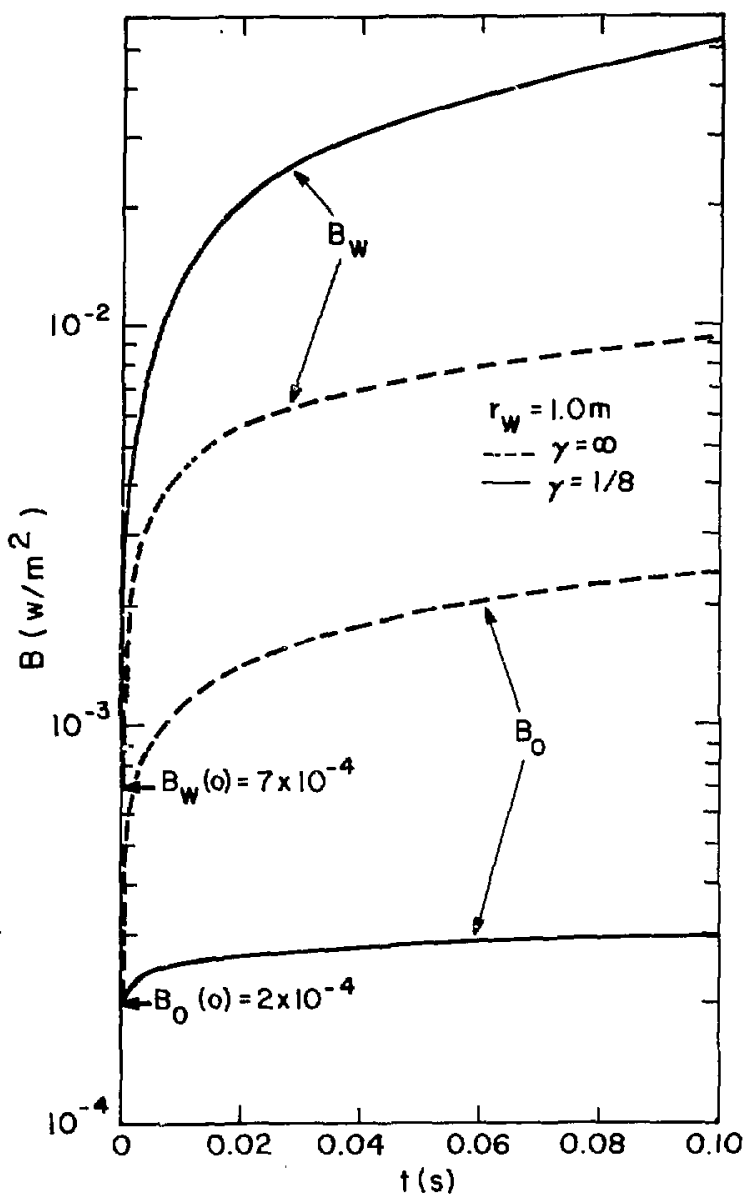

F1g. 11

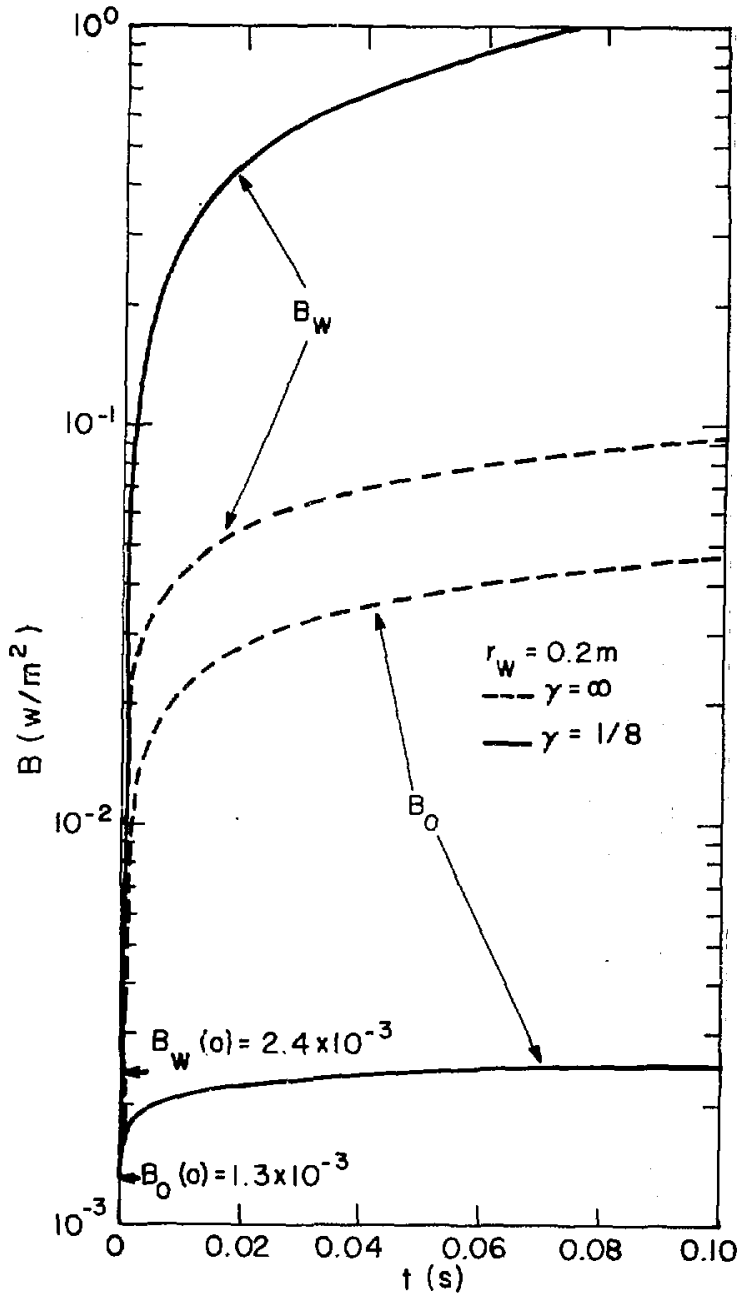

Fig. 12

Time history of the magnitude of the axial magnetic fleld at the flux conserving wall, $B_{w}$, and the magnitude rotating magnetic fleld, $B_{o}$, for $r_{w}=0.2 \mathrm{~m}$. 


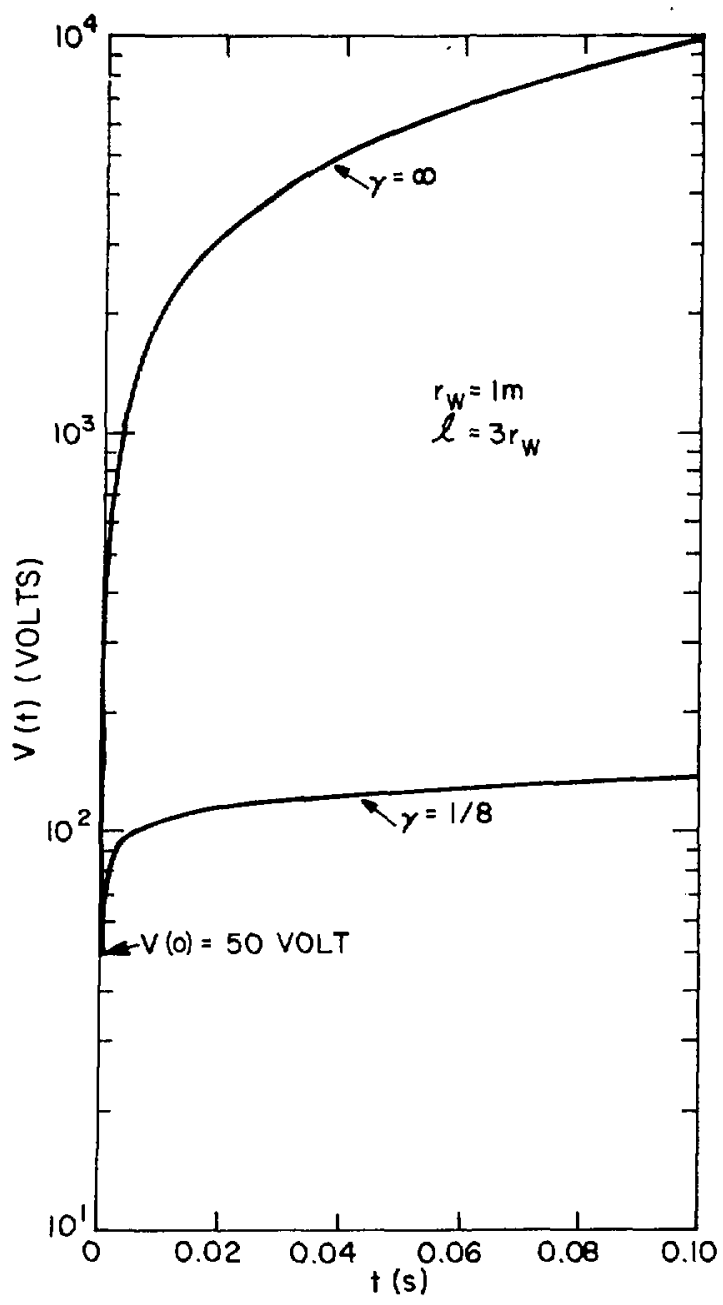

F1g. 13

Time history of the voltage applied to the dipole coll set that generates the rotating field for $r_{w}=1 \mathrm{~m}$ and $\ell=3 r_{w}$.

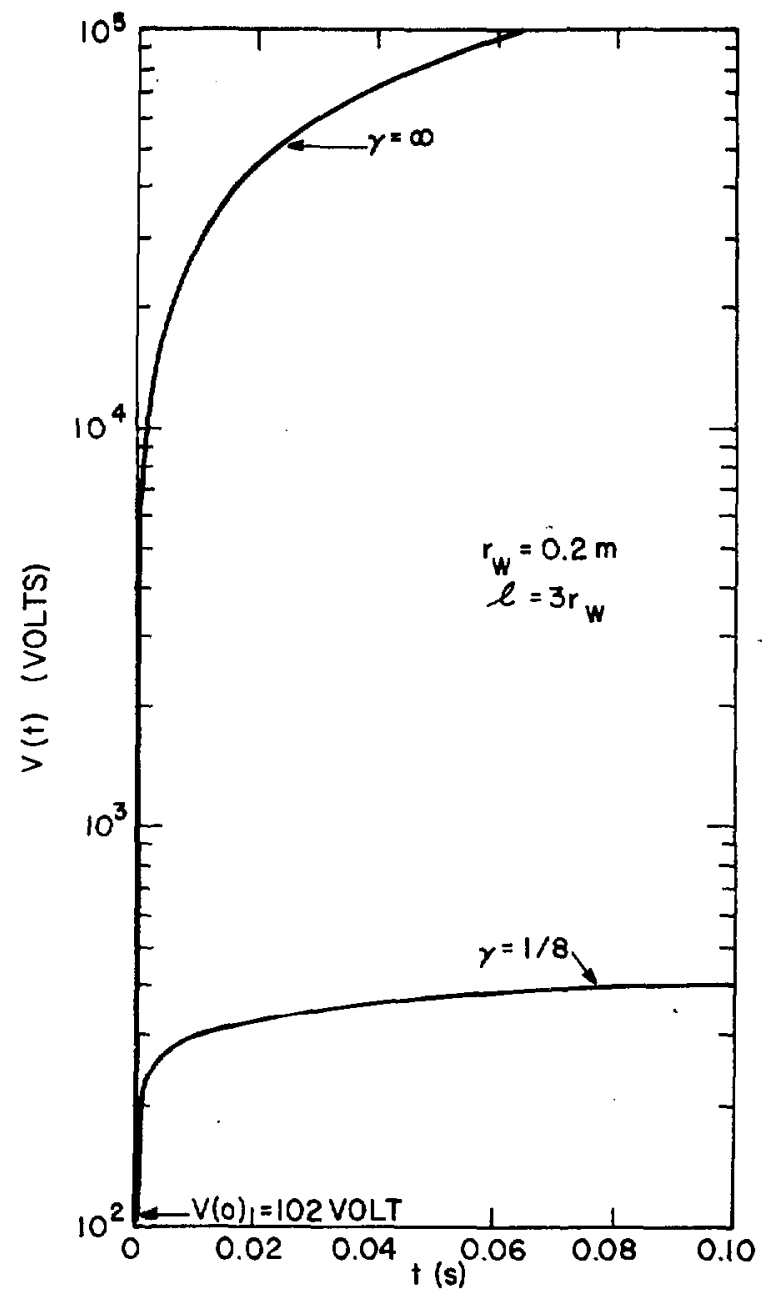

F1g. 14

Time history of the voltage applied to the dipole coll set that generates the rotating field for $r_{w}=0.2 \mathrm{~m}$ and $\ell=3 r_{w}$. 


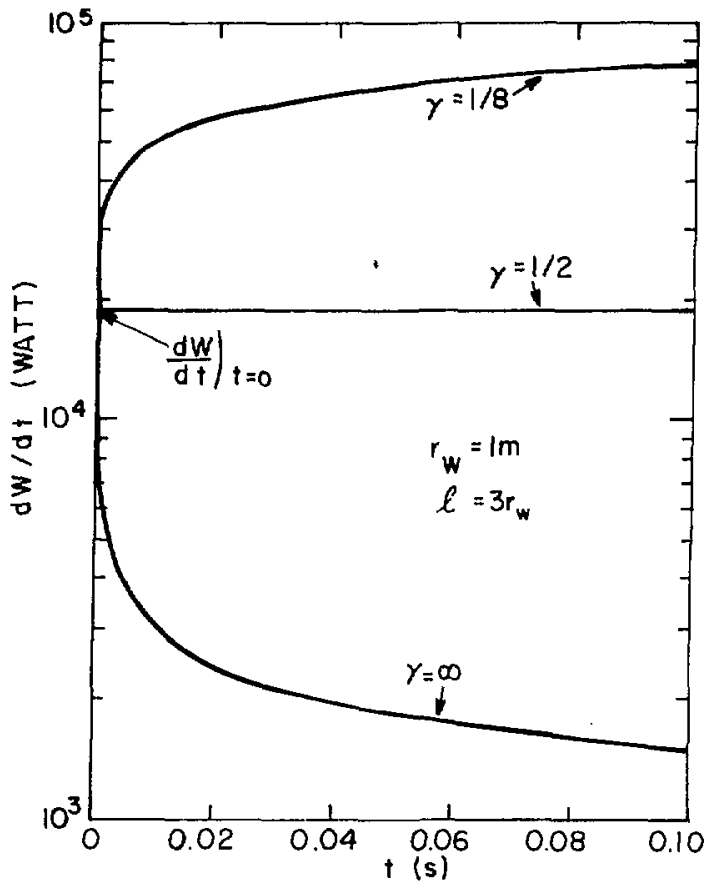

Fig. 15 Time history of the rate of energy deposition by ohmic heating into the electron plasma for $\mathbf{r}_{\mathbf{w}}=1 \mathrm{~m}$.

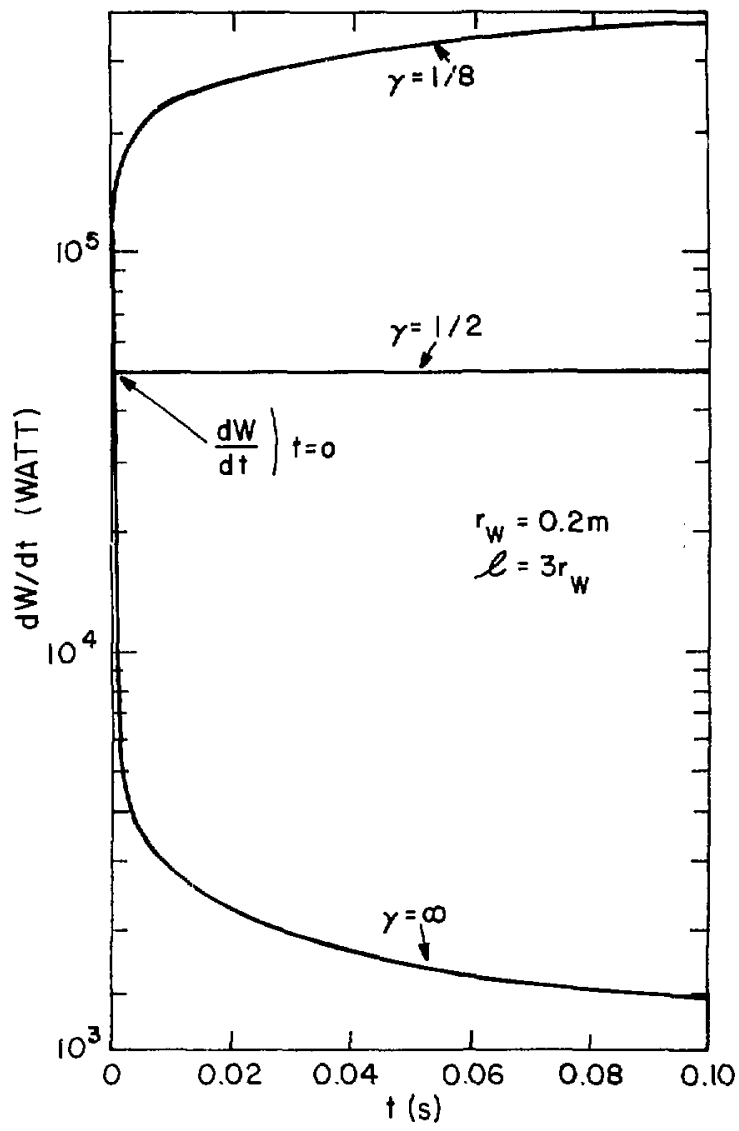

Fig. 16 Time history of the rate of energy deposition by ohmic heating into the electron plasma for $r_{w}=0.2 \mathrm{~m}$. 


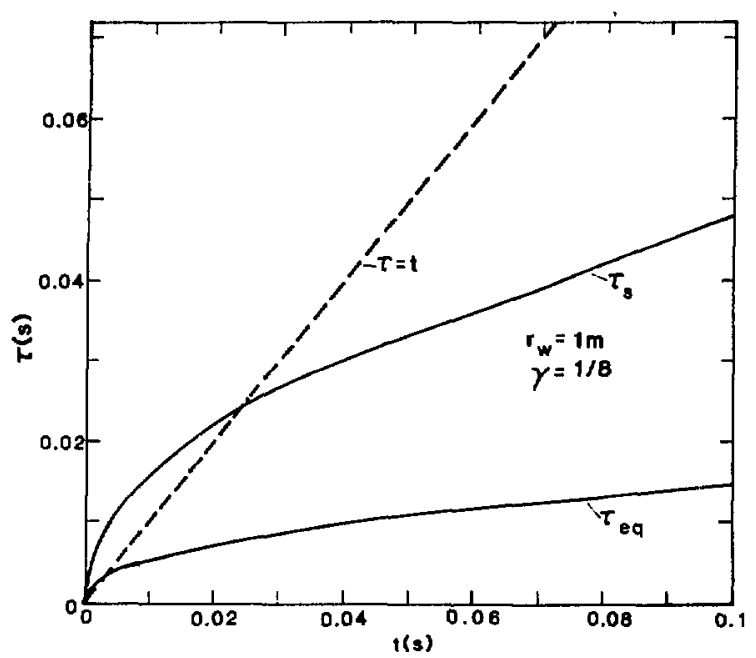

F1g. 17 Time history of the Ion spin-up time, $\tau_{8}$, and the electron-1on equilibration time $\tau$ eq, for $Y=1 / 8$ and $r_{W}=1 \mathrm{~m}$.

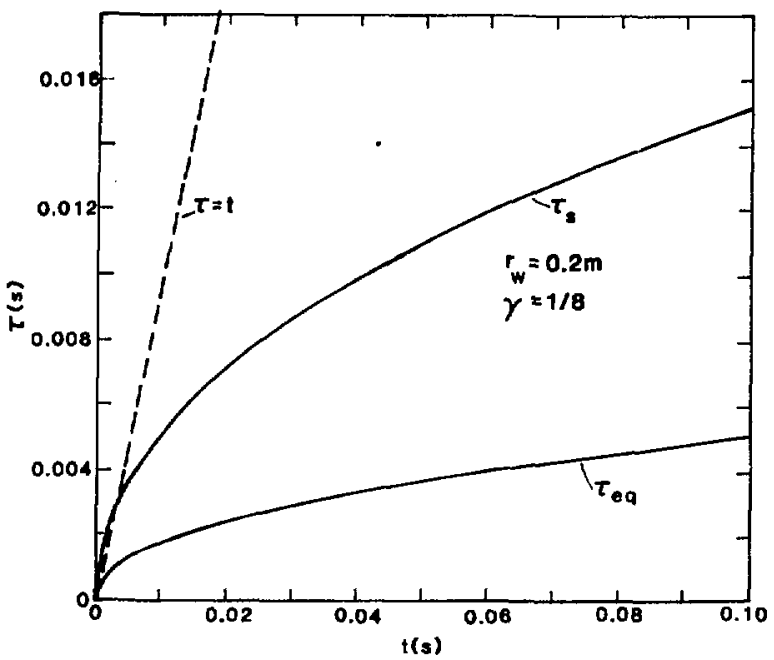

Fig. 18 Time history of the Ion spin-up time, $\tau_{s}$, and the electron-tion equilibration time, $\tau_{\text {eq, }}$ for $\gamma=1 / 8$ and $r_{w}=0.2 \mathrm{~m}$. 